\title{
Low-Value Recyclable Waste Regeneration in China: High-Efficiency Automatic Sorting Method and Experimental Research
}

\section{Tianchen Ji ( 541738243@qq.com )}

Huaqiao University - Xiamen Campus https://orcid.org/0000-0003-0722-0543

Huaiying Fang

Huaqiao University - Xiamen Campus

Rencheng Zhang

Huaqiao University - Xiamen Campus

Jianhong Yang

Huaqiao University - Xiamen Campus

Lulu Fan

Shenzhen Municipal Engineering Corporation

Jiantao Li

Huaqiao University - Xiamen Campus

\section{Research Article}

Keywords: Intelligent sorting, Low-value recyclable waste, Waste sorting, Process design

Posted Date: February 18th, 2022

DOI: https://doi.org/10.21203/rs.3.rs-1170355/v1

License: () (1) This work is licensed under a Creative Commons Attribution 4.0 International License.

Read Full License 


\section{Abstract}

The municipal domestic waste (MDW) generated in China is increasing year by year, but the waste that has been regenerated is very little. In order to efficiently sort low-value recyclable waste (LVRW), which is the largest recyclable part of MDW, we designed and manufactured a machine vision pneumatic sorting equipment with waste recognition accuracy higher than $92 \%$. Furthermore, an automated sorting method combining Mechanical sorting, near-infrared (NIR) sorting and machine vision sorting was developed specifically for MDW sorting. In field applications, the entire automated production line we built divides MDW (100\%) into six types (37.7\%) of single material waste and three types (33\%) of composite waste, which is very beneficial to the recycling of resources in the future.

\section{Introduction}

China, the world's most populous country, generated 235.6 million tons of municipal domestic waste (MDW) in only 196 large and medium-sized cities in 2020 (Ministry of Ecology and Environment of the People's Republic of China, 2020). Approximately $30 \%$ of the total waste is low-value recyclable waste (LVRW), including recyclable plastics, composite paper, glass, etc. (iiMedia.cn, 2019), but most of the LVRW is eventually incinerated and landfilled (Cheng et al., 2020; Duan et al., 2019). As shown in Fig. 1, MDW regeneration methods still account for a small proportion. And manual sorting, which has characteristics such as poor working environment, low efficiency, high uncertainty, and high wage costs, is still widely used in China. Recyclable waste (RW), especially LVRW, has the characteristics of large quantity, low recycling efficiency, and low profit, which requires centralised recycling and treatment to regain recycling value.

Therefore, automated waste sorting is the trend of MDW disposal. With the continuous progress of new sensor technology and artificial intelligence technology, MDW sorting methods are undergoing significant changes. Various MDW sorting methods and common sorting equipment are presented in Table 1.

Table 1 A summary of common MDW sorting methods (except manual sorting) and equipment. 
Sorting method Sorting basis

$\begin{array}{ll}\begin{array}{l}\text { Mechanical } \\ \text { sorting }\end{array} & \text { Based on the basic physical } \\ \text { properties of waste, such as } \\ \text { size, shape, density, etc. }\end{array}$

Electromagnetic
sorting

Based on the magnetic and electromagnetic property of waste

$\begin{aligned} & \text { Photoelectric } \\ & \text { sorting }\end{aligned}$
$\begin{aligned} & \text { Machine vision } \\ & \text { sorting }\end{aligned}$

Based on the optical properties of waste

Based on the image

characteristics (size, shape, colour, surface texture, contour, etc.) of waste
Common sorting equipment

Trommel screen, bounce screen, disc screen, vibrating screen, ballistic separator, winnowing machine, bag opener, flotation machine, etc.

Eddy current separator, magnetic separator, electrostatic separator, etc.

Near-infrared (NIR) sorting machine, colour sorter, etc.

Machine vision sorting machine

\subsection{Mechanical sorting methods}

Mechanical sorting technologies and equipment are mainly used to sort waste with different physical properties. Mechanical sorting methods are the most used methods in waste sorting for many years. According to the size, density, 2D, 3D shape, and other physical differences between different MDWs, the waste is separated using physical methods such as screening, flotation, and winnowing.

(Pongstabodee et al., 2008) successfully separated mixed post-consumer plastic waste (high-density polyethylene (HDPE), polypropylene (PP), poly ethylene terephthalate (PET), polyvinyl chloride (PVC), acrylonitrile butadiene styrene (ABS), and polystyrene (PS)) using a combination of the sink-float method and the selective flotation technique.

(Hansen et al., 2007) investigated the effect of screw press and disc screen pre-treatment technology on local municipal organic waste in Denmark.

(Ying and Cheng, 2011) developed a detailed theoretical design and calculation procedure of the shape and position of the blade of a bag breaker, which provides a theoretical reference for the research and development of related waste treatment equipment.

\subsection{Electromagnetic sorting methods}

Electromagnetic sorting methods are typically used to separate metals and plastics. Electromagnetic sorting methods mainly include magnetic sorting, electrostatic sorting, and eddy current sorting (Gundupalli et al., 2017). These methods are based on the magnetic difference, electrical difference of different components, and the principle of electromagnetic induction to separate waste.

(Aksa et al., 2013) developed a new mixed plastic particle (polycarbonate (PC) and polyamide (PA)) electrostatic separation craft that achieved $95 \%$ purity. 
(Maria and Lucian, 2011) introduced and analysed a combined model of probe coil metal parts based on eddy currents for conductive materials or ferrous metal/non-ferrous metal separation.

(Bonifazi and Serranti, 2012) introduced magnetic head pulley, belt magnet, drum magnet, magnetic drum separator, eddy current separator, electrostatic separator, and their applications in resource recycling.

\subsection{Photoelectric sorting methods}

Photoelectric sorting methods are usually used to distinguish waste of different colours or materials, such as plastic slices in different colours or boxes of different materials. Photoelectric sorting methods mainly use the surface light reflection effect of different objects and the difference in the reflection spectrum of the hydrogen-containing groups of organic molecules to identify materials.

(Wu et al., 2019) used multiple algorithms to analyze the Attenuated total reflection Fourier transform infrared spectroscopy acquired from textile samples, which achieved $100 \%$ accuracy in the classification test of 350 textile samples.

(Xiao et al., 2019) used Pythagorean wavelet transform to obtain the characteristic reflectivity of construction waste in the NIR band, and then used random forest and extreme learning machine to classify objects according to the characteristic reflectivity. They achieved $100 \%$ recognition accuracy for 180 objects in six categories including wood, plastics, bricks, concretes, rubbers, and black bricks.

(Rozenstein et al., 2017) tested the mid-wave infrared (MWIR, 3-12 $\mu \mathrm{m}$ ) spectral characteristics of black, coloured, and transparent PET, PE, PVC, PP, polylactic acid (PLA), and PS plastics. They compared the spectral characteristics of black, coloured, and transparent samples with results in literature.

Companies such as TOMRA Sorting GmbH (Balthasar, 2013; Harbeck, 2014; Klokkerud, 2011; Tomra, 2019), Sesotec GmbH (Sesotec, 2020), Steinert GmbH (Steinert, 2016), and Pellenc ST (Pellenc ST, 2019) have already developed NIR automatic sorting equipment, which have been used in many countries and regions in Europe, America, and Asia.

\subsection{Machine vision sorting methods}

Machine vision sorting methods mainly use deep learning algorithms to recognise waste with different colour, shape, position, surface texture, and other image characteristics. Machine vision sorting methods are the latest automated sorting technologies that have been developed rapidly with advancements in artificial intelligence neural networks.

(Zhihong et al., 2018) combined a visual inspection system with a robotic arm system, used deep learning algorithms to identify the position, posture, and type of target on the conveyor belt, and used KUKA robots to achieve waste classification. 
Companies such as BHS (Bulk Handling Systems, 2017), DataBeyond (Databeyond, 2019), and JONO (JONO ENVIRONMENTAL, 2020) have developed artificial intelligence sorting equipment based on machine vision, which are expected to be practically used in construction and demolition (C\&D) waste sorting plants.

These four types of sorting methods have different features and application scenarios.

- Mechanical sorting methods have a large production capacity and the lowest cost. Hence, they are widely used in front-end sorting of various wastes, but can only sort waste with differences in physical properties.

- Electromagnetic sorting methods can separate metals from the waste stream, or separate objects with different electrophysical properties. However, there are few types of waste that can be separated.

- Photoelectric sorting methods can sort objects of different materials or different colours more accurately. Among them, NIR sorting methods that can sort waste of different materials, find it difficult to classify waste of the same material, full label covered objects or black objects. And the equipment is expensive due to the high price of the NIR spectrometer.

- Machine vision sorting methods can classify waste in different image features with a lower cost than NIR sorting methods, but are unable to classify waste with similar image features.

To ensure the efficiency and generalizability of automatic sorting, the flexible combination of these four methods is necessary. In China, the number of automated MDW sorting centres is still relatively small, and there is no automatic sorting method especially for LVRW. Moreover, advanced automated sorting solutions in Europe and other places cannot be directly used in China due to the difference of MDW characteristics such as composition, proportion, and oil content. Therefore, we aim to design a MDW sorting method that is suitable for the LVRW status in China.

In this study, we designed and manufactured a machine vision sorting equipment, and proposed a specialized automatic sorting method for LVRW, combining Mechanical sorting, NIR sorting, and machine vision sorting (mechanical-NIR-vision sorting method). Finally, experiments were conducted to test the feasibility and efficiency of the machine vision sorting equipment, and the entire automatic sorting method. The experimental results reveal that our method can initially achieve a waste reduction of $37.7 \%$, and a waste reduction of more than $70.7 \%$ that can be achieved.

\section{Materials And Methods}

\subsection{Experimental materials}

The waste used in the experiment was unsorted MDW provided by waste collection sites in Xiamen City, Fujian Province, China, and had the following characteristics: 
The main components of waste were paper, PET, PP, PS, PVC, EPS, LDPE, HDPE, polyvinyl alcohol (PVA), aluminium, and iron. Owing to the low source separation level (SSL), RW was mixed with organic waste and non-recyclable waste, which were classified as "other material". In order to simplify the calculation, we used the main material as the mixed material classification basis to classify the waste, and waste with a quality percentage less than $0.1 \%$ was classified as "other material".

The material composition, quality percentage, and price of different wastes are listed in Table 2.

Table 2 Material composition, quality percentage and price of different wastes.

\begin{tabular}{llll} 
Material & Common waste & Quality percentage (\%) & Price (Yuan/ton) \\
\hline Paper & Ordinary paper & 8 & 800 \\
& Composite paper & 7 & 400 \\
\hline Plastic & Plastic film & 20 & 600 \\
& Colour PP box & 10 & 2200 \\
& PET blister tray & 8 & 900 \\
& Transparent PP box & 6 & 2900 \\
& Foam board & 4 & 2800 \\
& Miscellaneous plastic & 4 & 1000 \\
& PET bottle & 1.7 & 2950 \\
\hline Metal & Iron & 1.2 & 1900 \\
& Aluminum can & 0.1 & 7500 \\
\hline Other & Post-sorting waste & 13 & -50 \\
\hline & Pre-sorting waste & 11 & -50 \\
\hline & Bulky waste & 6 & uncertain
\end{tabular}

\subsection{Design of machine vision sorting equipment}

In order to realize the effective sorting of the entire sorting plant, we need to ensure the sorting efficiency of a single equipment first. To make up for the disadvantages of NIR sorting equipment, we designed and manufactured a machine vision pneumatic sorting equipment with visual inspection technologies, and it is a good supplement to overcome the limitations of NIR spectroscopy detection.

\subsubsection{Component description of machine vision sorting equipment}


The equipment hardware structure is shown in Fig. 2, which is mainly composed of four parts: (a) highspeed conveyor belt, (b) image acquisition system, (c) industrial computer, and (d) sorting actuator.

(a) High-speed conveyor belt: A high-speed conveyor belt with a speed greater than $3 \mathrm{~m} / \mathrm{s}$ is mainly used for the transportation and distribution of objects. The unsorted waste falls from a high position above the belt to achieve high horizontal and vertical relative speeds on the conveyor belt, due to which the objects bounce on the belt, collide with each other, and then pull apart.

(b) Image acquisition system: An image acquisition system is used, which is located above the end of the high-speed conveyor belt. It consists of a detection cage containing a colour CMOS line scan camera (DALSA, Canada) and a high-brightness coaxial light source (OPT, China). The clear MDW images can be collected at high speed and sent to the industrial computer in real time to detect waste information.

(c) Industrial computer: An industrial computer (Advantech, Taiwan) with a graphic processing unit (NVIDIA, USA) receives the MDW images collected by the image acquisition system. The pre-trained deep learning network is used for detection after preprocessing, and then the information of each object is obtained to determine the control logic of the actuator.

(d) Sorting actuator: For the MDW sorting actuator, solenoid valves (MAC, America) are used, which are arranged side by side to form a valve group. These valves can eject gas at pressure up to 6 bar. When the target object passes over the air outlet, the industrial computer program controls the solenoid valves in the corresponding position to open through the programmable logic controller (SIEMENS, Japan). Highpressure gas is discharged to blow the material to a far place, in order to classify the target objects that are blown out to the far ends and the non-target objects that are tossed to the near point obliquely.

\subsubsection{Operational mechanism of machine vision sorting equipment}

The equipment software logic is shown in Fig. $\mathbf{3}$, which is mainly composed of (a) the image detection algorithm and (b) the valve control algorithm.

(a) Image detection algorithm: In order to ensure the accuracy of the valve injection position and the efficiency of the detection algorithm, it is necessary to predict the type, position, and contour information of the object accurately and quickly. Therefore, we selected the Mask R-CNN (Fig. 4) instance segmentation network with ResNet50-D (He et al., 2018) as the backbone. In order to adapt to changes in the shape and pose of the object, Deformable ConvNets v2 (DCNv2) (Zhu et al., 2018) is partly used in the backbone. Then, 10,000 high-definition MDW images (Fig. 5) were captured and labeled to train the neural networks to obtain the prediction model, which was used to predict the image data from the image acquisition system and return the result we need in real time.

(b) Valve control algorithm: According to the information output by the image detection algorithm, the compiled program can calculate the ejection time when the object moves above the valve group and the serial numbers of the valves directly below the object. When an object passes over the valve, the compiled 
program controls the programmable logic controller to open the valve of the corresponding position, and sprays high-pressure gas to blow out the target object.

\subsubsection{Generation of LVRW prediction model}

In order to ensure the waste recognition accuracy of the image detection algorithm and save the cost of manual labeling, we generated a LVRW prediction model to identify and locate the waste in the image as follows (Fig.6).

First, 2000 pieces of high-definition MDW images were labeled with polygon labels to train for a semiautomatic annotation model. Then, the model was used to predict the pseudo-labels of the objects in the rest of 8000 MDW images and corrected them manually. Finally, the 10000 labeled images were mixed, and using data augmentation methods like horizontal/vertical flip, rotate, affine transformation and random brightness to get an enlarged dataset with 30000 labeled images. The final prediction model was trained on the 30000 labeled images and used for the actual prediction.

\section{3 Design of LVRW automatic sorting method}

When RW of different colours or materials are mixed to generate regenerated materials, the performance of the material deteriorates, and even RW of the same material and colour has different performance ratings. To finely classify different types of waste, an automatic sorting system was designed and built and we named it mechanical-NIR-vision sorting method.

The material transportation of the entire automatic sorting production line is mainly completed by conveyor belts. Manual sorting and feeding between various equipment are carried out through low-speed conveyor belts with a speed lower than $0.5 \mathrm{~m} / \mathrm{s}$. Pneumatic sorting equipment uses a high-speed conveyor belt with a speed greater than $3 \mathrm{~m} / \mathrm{s}$ to ensure that waste can be evenly dispersed on the conveyor belt without the help of front-end bulk devices to handle LVRW with large numbers and low density.

The specific sorting steps are shown in Fig. 7.

(a) Pre-sorting step: The first step of the method is to split the bagged waste into bulk. Before the waste enters the bag opener, we manually sort the waste that is overly large or unsuitable for entering the sorting line, such as home appliances and metal buckets, to avoid blockage and equipment damage. After selection, the waste enters the bag opener, which is composed of two rollers that have blades on the surfaces and rotate in opposite directions. The bags are cut while ensuring that the waste in the bags is relatively intact to realise the separation of the bagged waste.

(b) Waste separation by size: The waste after bag breakage is sent to a variable-aperture trommel by a conveyor belt. The small holes first screen out pre-sorting waste, such as fine dirt and small objects. Then, the waste that is suitable for sorting, is screened out of the medium-sized holes. Finally, the foam board and bulky waste with a volume larger than the sortable size are ejected from the discharge port of the 
drum screen, and then sorted manually according to the material. In this phase, the foam board is picked out.

(c) Separation of paper: Waste paper is mainly divided into composite paper and ordinary paper. Compared with the physical characteristics and image characteristics, the NIR signature of paper are distinct from those of other wastes. Therefore, NIR pneumatic sorting equipment is used to separate out paper. Composite paper such as Tetra Pak packages are composite material composed of paper, aluminium, and plastic. The regeneration of composite paper requires special separation processing technology to separate the materials in order to obtain higher recycling value. NIR sorting equipment can distinguish different types of paper through the reflection spectra of composite materials and single material. Therefore, NIR pneumatic sorting equipment is used to separate composite paper and ordinary paper.

(d) Separation of 2D and 3D waste: The waste stream after removing paper, mainly contains plastic films, bottles, food boxes, metal cans, and other debris. Among them, plastic films are thin, flat 2D objects, and other materials are heavy 3D objects and hence, can be easily scrolled. Thus, a bounce screen is chosen to separate the waste stream. The eccentric shaft can drive the inclined sieve plate for reciprocating motion and transports the waste falling into the middle of the sieve plate upwards. 2D objects are transported to the top of the bounce screen by friction, 3D objects are rolled down to the bottom of the bounce screen, and the scraps produced by the sorting, leak out from the screen holes. The 2D flat objects on the sieve are separated by NIR pneumatic sorting equipment to separate the plastic film from the objects of other materials.

(e) Separation of plastic in 3D waste: Among the 3D waste sifted down by the bounce screen, plastic waste, which is mainly PP and PET waste, accounts for a large proportion. In PP waste, the recycling value of transparent PP waste, such as transparent PP food boxes and transparent PP cups is higher than other colours; in PET waste, the recycling value of PET bottles is high, and the recycling value of PET blister trays such as PET food boxes and PET trays is relatively lower. However, transparent PET blister trays and transparent PP boxes are similar in appearance and shape. Therefore, waste can't be classified by machine vision. Hence, NIR pneumatic sorting equipment is first used to blow out the transparent PP waste in $3 \mathrm{D}$ waste, then machine vision pneumatic sorting equipment is used to separate colour PP waste (white, black, red, grey, yellow, etc.) according to the colour and shape characteristics. When recycling PET in the waste stream after the separation of PP waste, a machine vision pneumatic sorting equipment is used to blow out PET waste. Machine vision pneumatic sorting equipment is still used to finely classify of PET waste by identify the image characteristics of PET bottles and PET blister trays.

\section{Results And Discussion}

To verify the performance of the machine vision sorting equipment and the feasibility of the mechanicalNIR-vision sorting method, in this study, we first tested the recognition accuracy of the target detection algorithm. Then we collaborated with Luhai Pro-environment Co., Ltd. to build an automated MDW 
sorting plant to dispose local MDW in Xiamen City, Fujian Province, according to the designed MDW sorting method shown in Fig. 7. The composition of the MDW is listed in Table 2. To explore the effect of machine vision sorting and the entire method in the refined separation of LVRW in Xiamen City, we tested two sorting methods: the mechanical-NIR sorting method and the mechanical-NIR-vision sorting method. And the field production test was done finally.

\subsection{Target detection accuracy of machine vision sorting equipment}

The sorting accuracy of machine vision sorting equipment is mainly composed of target detection accuracy and actuator sorting accuracy. In this section, we only evaluated the target detection accuracy by using precision (the number of correctly classified samples in the class / the total number of samples classified into this class) and recall (the number of correctly classified samples in the class / the total number of samples in the class). The detection method involved placing the mixed MDW directly on the high-speed conveyor belt, and then using the image detection program to collect, predict, and save the image marked with the prediction results. For the result calculation, we artificially counted and calculated the true labels and predicted labels of the objects in the saved images with a confidence $>0.75$.

The confusion matrix of the image detection test result is shown in Fig. $\mathbf{8}$.

It can be observed from the confusion matrix that the recognition precision of most objects is higher than 92\%. However, the similar features between transparent PP box and PET blister tray cause low precision and recall. In field applications, NIR sorting is used to sort waste mixed with a variety of transparent materials to compensate for the inability to obtain higher accuracy through machine vision sorting. Thr actual practical image detection accuracy is higher than that shown in Fig. $\mathbf{8}$.

\subsection{Mechanical-NIR sorting method}

In the mechanical-NIR sorting method, we replaced the steps originally designed using machine vision sorting with NIR sorting to test the sorting effect, and as a result, some of the sorting results are not satisfactory. The sorting results are shown in Fig. 9.

The experimental results show that:

(a) \& (b) It is difficult for NIR sorting method to distinguish waste with different characteristics but with same material.

(c) NIR sorting method can't identify the true material of waste which covered by label.

(d) NIR sorting method is unable to detect dark objects.

\subsection{Mechanical-NIR-vision sorting method}

The mechanical-NIR-vision sorting method shown in Fig. 7 was tested and compared with the mechanical-NIR sorting method. The sorting results in Fig. 10 (a), (b), (c) and (d) correspond to those in 
Fig. 9 (a), (b), (c) and (d) respectively.

The experimental results show that machine vision sorting is a suitable supplement to NIR sorting. In machine vision sorting, a model with generalised performance is obtained through the labeled image data set, in order to "memorise" and identify different objects, it is suitable for the classification of waste with different appearances, and dark objects also have observable features in the image.

\subsection{Final sorting result}

Eventually, the entire production line separates the following nine types of RW: bulky waste, foam board, ordinary paper, composite paper, plastic film, transparent PP box, colour PP box, PET bottle, and PET blister tray. The final sorting results are shown in Fig. 11.

The precision of the sorted waste is directly related to the complexity of the subsequent recycling process. If the precision of sorting is sufficiently high during front-end sorting, even part of the process in recycling can be reduced. The foam board, ordinary paper, transparent PP box, colour PP box, PET bottle, and PET blister tray that were sorted in the final sorting test were all single material with a purity of more than $92 \%$, which helped remove the first sorting step in the regeneration process to save cost and improve efficiency.

Bulky waste, large waste and plastic film have complex components and should be subdivided into different materials. Composite paper and part of the plastic film are mixed materials. Therefore, they need to be further separated to increase the recycling value. For miscellaneous plastic and metal, manual sorting was used. Pre-sorting waste and post-sorting waste are scraps that cannot be recycled and are generally incinerated or landfilled.

The entire production line is mainly aimed at recycling LVRW, which has been gradually emphasised in China in recent years. The production capacity of the MDW automatic sorting plant developed in this study can reach 45 tons/day, which can initially achieve a reduction of $37.7 \%$ (foam board $4 \%$, ordinary paper $8 \%$, transparent PP box $6 \%$, colour PP box $10 \%$, PET bottle $1.7 \%$, and PET blister tray $8 \%$ ). If we continue to finely classify composite waste and bulky waste, it is possible to achieve a waste reduction of more than $70.7 \%$ (bulky waste $2 \%$, large waste $4 \%$, composite paper $7 \%$ and plastic film $20 \%$ ).

In this section, we first verified the reliability of machine vision sorting, and then tested the field application effect of the mechanical-NIR sorting method and the mechanical-NIR-vision sorting method. It is concluded that NIR and machine vision can complement each other and achieve accurate classification of LVRW. Compared with manual sorting, the mechanical-NIR-vision sorting method used in this study is more efficient, has a better working environment, and lower labour costs. Compared with the mechanical-NIR sorting method commonly used in, our sorting method has a better adaptability and universality for the sorting of LVRW in China.

\section{Conclusion}


In this study, a machine vision pneumatic sorting equipment was designed and manufactured. Moreover, a mechanical-NIR-vision sorting method was designed and built in Xiamen specifically for LVRW sorting. Then we designed experiments and verified that the combination of NIR sorting and machine vision sorting is more suitable for local MDW treatment. For machine vision sorting equipment, the sorting accuracy is higher than $92 \%$. And the entire automated production line divides MDW into six types (37.7\%) of single material waste that can directly enter the regeneration process, and three types $(33 \%)$ of composite waste and mixed waste that require further classification or dissociation to be regenerated, which demonstrates the effectiveness of the equipment we developed and method combining NIR sorting and machine vision sorting for sorting MDW.

Miscellaneous plastic and metal waste can be sorted by machine vision parallel robots, but they account for a relatively small proportion, and this part is not yet automated. The recognition and sorting of plastic film and paper have not yet been tested, which is the key part of our research in the future.

Nonetheless, the method in this article is the first known solution for automatic sorting of LVRW in China, and it will continue to be improved and promoted for various applications to further contribute to the reduction of waste.

\section{Declarations}

Conflict of interest The authors declare that they have no conflict of interest.

Authors' contributions Tianchen Ji: Methodology, Writing - Original Draft, Investigation. Huaiying Fang: Writing - Review \& Editing, Resources. Rencheng Zhang: Conceptualization, Supervision.

Jianhong Yang: Project administration, Funding acquisition. Lulu Fan: Formal analysis, Visualization, Data curation. Jiantao Li: Software, Validation.

Funding This research was financially supported by the Major Program of Industry and University Cooperation of Fujian Province (2020H6012), the Major Special Program of Science and Technology of Fujian Province (2020YZ017022), and the Key Technologies Research and Development Program of Shenzhen (JSGG20201103100601004).

Data availability The datasets generated during and/or analyzed during the current study are available from the corresponding author on reasonable request.

\section{References}

1. Aksa, W., Medles, K., Rezoug, M., Boukhoulda, M.F., Bilici, M., Dascalescu, L., 2013. Two stage electrostatic separator for the recycling of plastics from waste electrical and electronic equipment. $J$ ELECTROSTAT 71(4), 681-688.

2. Balthasar, D., 2013. Apparatus, system and method for detecting matter. 
3. Bonifazi, G., Serranti, S., 2012. Recycling Technologies.

4. Bulk Handling Systems, 2017. BHS AND NRT INTRODUCE MAX-AI ${ }^{\text {TM }}$ TECHNOLOGY. https://www.maxai.com/.

5. Cheng, J., Shi, F., Yi, J., Fu, H., 2020. Analysis of the factors that affect the production of municipal solid waste in China. J CLEAN PROD 259, 120808.

6. Databeyond, 2019. PiCKiNG $\cdot \mathrm{Ai}^{\mathrm{TM}}$ Achieving smarter sorting through "self-learning".

7. Duan, H., Song, G., Qu, S., Dong, X., Xu, M., 2019. Post-consumer packaging waste from express delivery in China. Resources, Conservation and Recycling 144, 137-143.

8. Gundupalli, S.P., Hait, S., Thakur, A., 2017. A review on automated sorting of source-separated municipal solid waste for recycling. WASTE MANAGE 60, 56-74.

9. Hansen, T.L., Jansen, J., Davidsson, A., Christensen, T.H., 2007. Effects of pre-treatment technologies on quantity and quality of source-sorted municipal organic waste for biogas recovery. WASTE MANAGE 27(3), 398-405.

10. Harbeck, H., 2014. INSPECTION APPARATUS.

11. He, T., Zhang, Z., Zhang, H., Zhang, Z., Xie, J., Li, M., 2018. Bag of Tricks for Image Classification with Convolutional Neural Networks.

12. iiMedia.cn, 2019. 2019-2021 China's Renewable Resources Industry Trends and Trends Analysis Report. https://www.iimedia.cn/c400/66825.html. (Accessed 2021/7/27 2021).

13. JONO ENVIRONMENTAL, 2020. Light Intelligent Sorting Robot- JONO ENVIRONMENTAL. https://www.jonochina.com/Al-SORTING/195.html.

14. Klokkerud, A., 2011. AN APPARATUS AND METHOD FOR INSPECTING MATTER.

15. Maria, B., Lucian, M., 2011. Analytical Model Of Eddy Current-based Device Meant To The Conductive Materials Sorting,Proceedings 25th European Conference on Modelling and Simulation.

16. Ministry of Ecology and Environment of the People's Republic of China, 2020. 2020 Annual Report on the Prevention and Control of Environmental Pollution by Municipal Solid Waste in Large and Medium-sized Cities.

17. Pellenc ST, 2019. The multi-material sorting machine for sorting and recycling centers - Pellenc ST. https://www.pellencst.com/products/mistral-connect-2/. (Accessed 2021-8-5.

18. Pongstabodee, S., Kunachitpimol, N., Damronglerd, S., 2008. Combination of three-stage sink-float method and selective flotation technique for separation of mixed post-consumer plastic waste. WASTE MANAGE 28(3), 475-483.

19. Rozenstein, O., Puckrin, E., Adamowski, J., 2017. Development of a new approach based on midwave infrared spectroscopy for post-consumer black plastic waste sorting in the recycling industry. WASTE MANAGE 68, 38-44.

20. Sesotec, 2020. Recycling sorting systems with conveyor belt. https://www.sesotec.com/emea/en/products/groups/recycling-sorting-systems-with-conveyor-belt. (Accessed 2020-11-18 2020). 
21. Steinert, 2016. UniSort BlackEye - sorting of bulk materials. https://steinertglobal.com/us/magnetssensor-sorting-units/sensor-sorting/nir-sorting-systems/unisort-blackeye. (Accessed 2020-11-18.

22. Tomra, 2019. Solutions for metal and waste sorting. https://www.tomra.com/en/sorting/recycling/tomra-solutions.

23. Wu, X., Li, J., Yao, L., Xu, Z., 2019. Auto-sorting commonly recovered plastics from waste household appliances and electronics using near-infrared spectroscopy. J CLEAN PROD.

24. Xiao, W., Yang, J., Fang, H., Zhuang, J., Ku, Y., 2019. A robust classification algorithm for separation of construction waste using NIR hyperspectral system. WASTE MANAGE 90, 1-9.

25. Ying, Q., Cheng, X.Y., 2011. A Study of Bag-Breaking Device Based on Whitworth Mechanism. Applied Mechanics and Materials 130-134, 1137-1141.

26. Zhihong, C., Hebin, Z., Yan, W., Yanbo, W., Binyan, L., 2018. Multi-task Detection System for Garbage Sorting Base on High-order Fusion of Convolutional Feature Hierarchical Representation. Technical Committee on Control Theory, Chinese Association of Automation, pp. 5426-5430.

27. Zhu, X., Hu, H., Lin, S., Dai, J., 2018. Deformable ConvNets v2: More Deformable, Better Results.

\section{Figures}

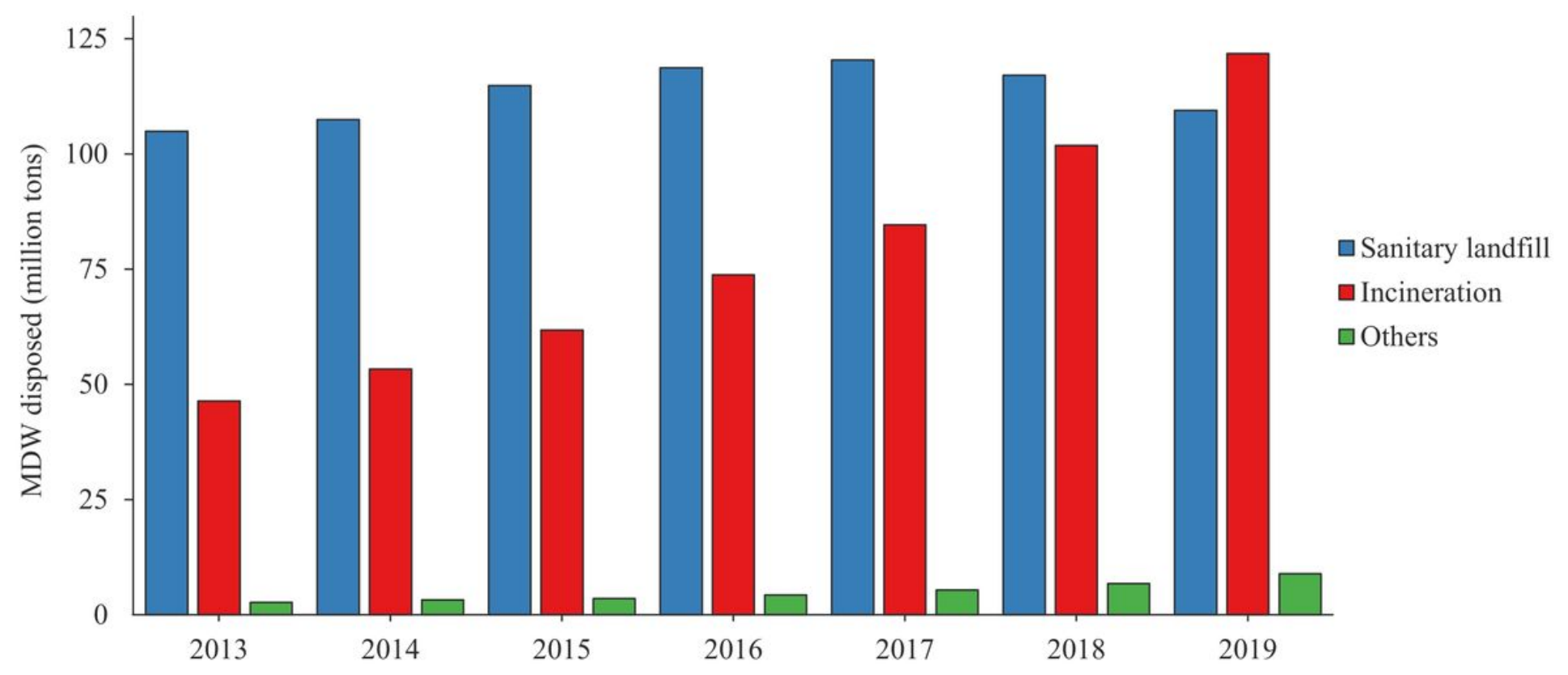

\section{Figure 1}

Annual disposal volume of different MDW disposal methods. 


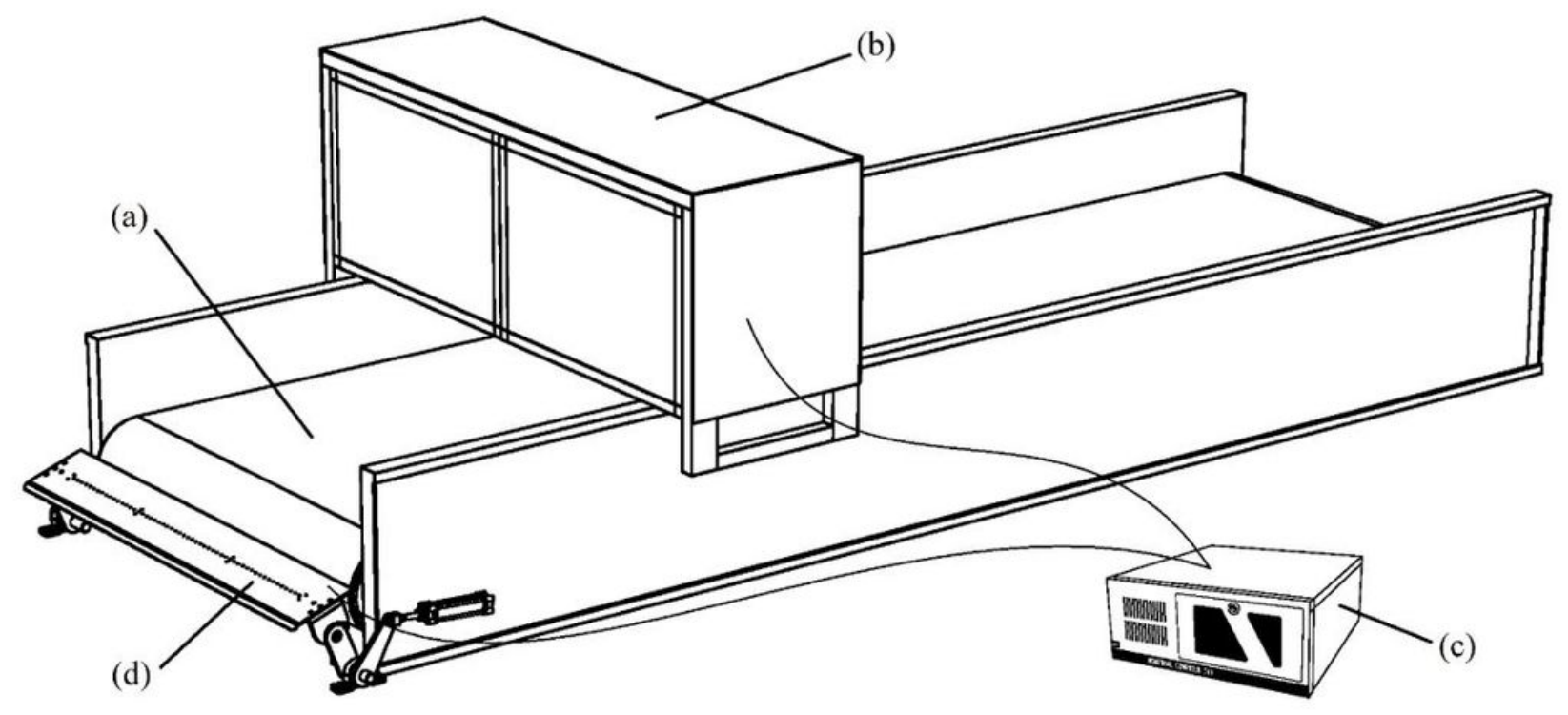

Figure 2

Equipment hardware design. (a) High-speed conveyor belt, (b) image acquisition system, (c) industrial computer, (d) sorting actuator.

Figure 3

Control algorithm flow.

Figure 4

Mask R-CNN network structure.

\section{Figure 5}

High-definition images of MDW.

\section{Figure 6}

Generation of LVRW prediction model. 
Figure 7

High-efficiency LVRW automatic sorting method.

\section{Predicted samples}

\begin{tabular}{|c|c|c|c|c|c|c|c|c|c|c|}
\hline & $\begin{array}{c}\text { Colour } \\
\text { PP box }\end{array}$ & $\begin{array}{c}\text { Black } \\
\text { PP box }\end{array}$ & $\begin{array}{c}\text { Trans } \\
\text { PP box }\end{array}$ & $\begin{array}{c}\text { Miscell- } \\
\text { aneous } \\
\text { plastic }\end{array}$ & $\begin{array}{c}\text { White } \\
\text { PP box }\end{array}$ & $\begin{array}{c}\text { PET } \\
\text { Bottle }\end{array}$ & $\begin{array}{c}\text { PET } \\
\text { blister } \\
\text { tray }\end{array}$ & Others & $\begin{array}{c}\text { Back } \\
\text { ground }\end{array}$ & Recall \\
\hline $\begin{array}{c}\text { Colour } \\
\text { PP box }\end{array}$ & 48 & 1 & 1 & 0 & 0 & 0 & 1 & 2 & 0 & 0.906 \\
\hline $\begin{array}{c}\text { Black } \\
\text { PP box }\end{array}$ & 0 & 77 & 1 & 0 & 0 & 0 & 0 & 1 & 1 & 0.963 \\
\hline $\begin{array}{c}\text { Trans } \\
\text { PP box }\end{array}$ & 3 & 4 & 158 & 1 & 0 & 0 & 3 & 10 & 5 & 0.859 \\
\hline Miscell- & & & - & & - & & & $\ldots$ & & $\ldots$
\end{tabular}

Figure 8

Confusion matrix of image recognition results. 


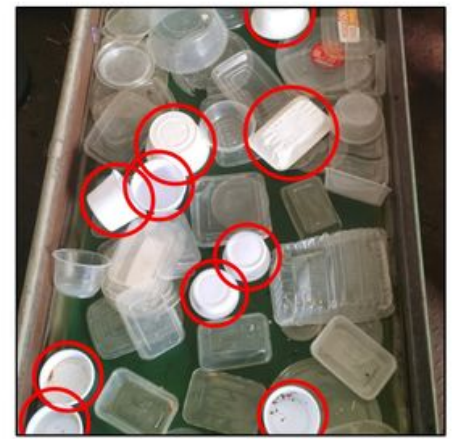

(a)

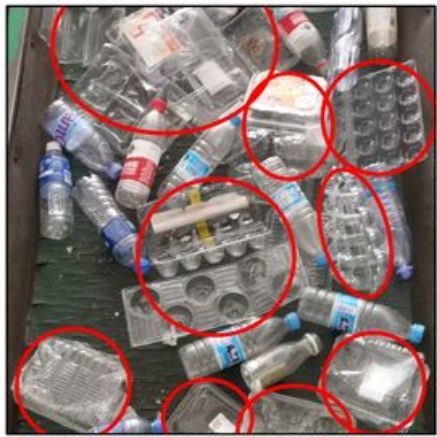

(b)

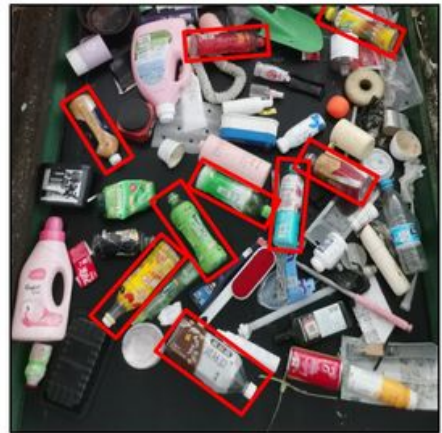

(c)

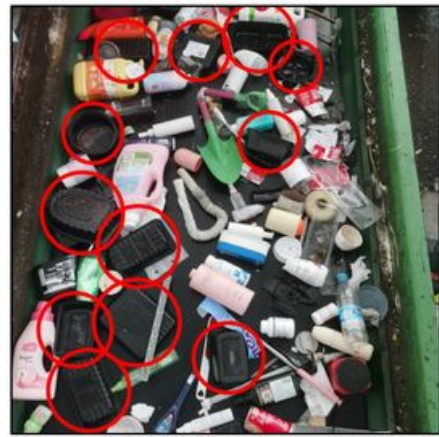

(d)

Figure 9

Results of NIR sorting method. (a) White PP boxes are ejected erroneously, (b) PET blister trays are brought out with PET bottles by mistake, (c) PET bottles covered with full labels are not sorted, (d) black PP boxes are not sorted.

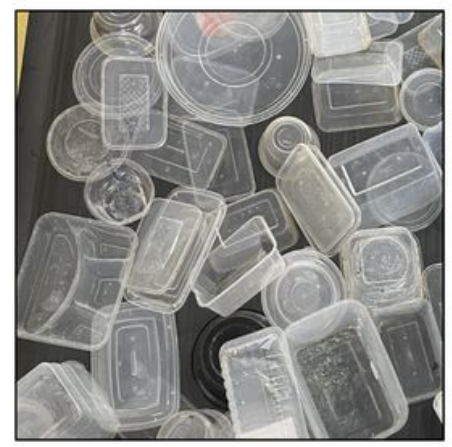

(a)

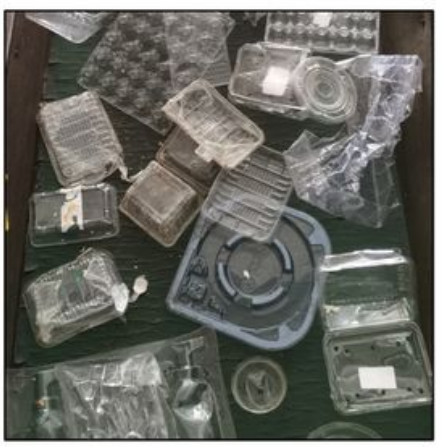

(b)

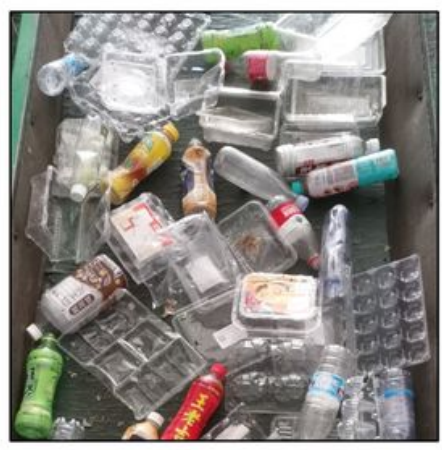

(c)

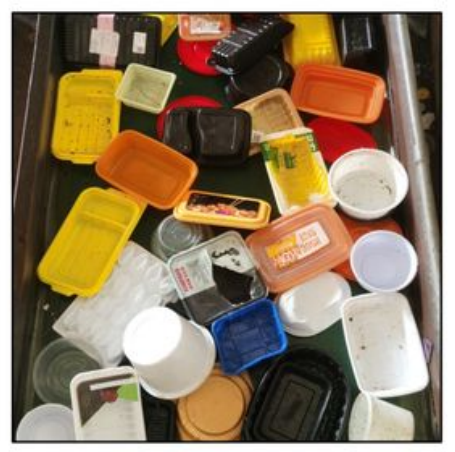

(d)

Figure 10

Results of machine vision sorting method. (a) White PP boxes are not ejected mistakenly, (b) PET blister trays are sorted to the right place, (c) PET bottles covered with labels are correctly sorted as PET waste, (d) Black PP boxes are correctly sorted as colour PP boxes.

\section{Figure 11}

Mechanical-NIR-vision sorting method results. (a) Bulky waste, (b) foam board, (c) ordinary paper, (d) composite paper, (e) plastic film, (f) transparent PP box, (g) colour PP box, (h) PET bottle, (i) PET blister tray.

\section{Supplementary Files}


This is a list of supplementary files associated with this preprint. Click to download.

- GraphicalAbstract.jpg

- Introductionvideo.mp4 